International Journal of Pure and Applied Mathematics

Volume 106 No. 3 2016, 753-758

ISSN: 1311-8080 (printed version); ISSN: 1314-3395 (on-line version)

url: http://www.ijpam.eu

doi: 10.12732 /ijpam.v106i3.5

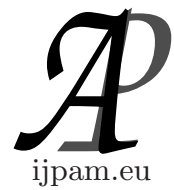

\title{
EVEN VERTEX COLORING OF A GRAPH
}

\author{
P. Siva Kota Reddy ${ }^{1 \S}$, Kavita S. Permi ${ }^{2}$ \\ ${ }^{1}$ Department of Mathematics \\ Siddaganga Institute of Technology \\ B.H. Road, Tumkur, 572 103, INDIA \\ ${ }^{2}$ Department of Mathematics \\ Acharya Institute of Technology \\ Bangalore, 560 107, INDIA
}

\begin{abstract}
As a generalization of Harary's notion of consistency in marked graphs, we define define an even vertex coloring of a graph $G$ as an assignment of colors to the vertices of $G$ such that in every cycle of $G$ there is a nonzero even number of vertices of at least one color. The even vertex coloring number $\varepsilon_{v}(G)$ of even-vertex colorable graph $G$ is defined as the minimum number of colors in an even vertex coloring of $G$ and a minimum even vertex coloring of $G$ is is one which uses exactly $n=\varepsilon_{v}(G)$ colors. A characterization of minimally edge-colored graphs is obtained and a result linking the notion to bipartite Eulerian multigraphs is established.
\end{abstract}

AMS Subject Classification: $05 \mathrm{C} 22$

Key Words: coloring, marked graphs, consistency, even vertex coloring

\section{Introduction}

For all terminology and notation in graph theory we refer the reader to consult any one of the standard text-books by Chartrand and Zhang [4], Harary [7] and West [8].

In this paper, unless mentioned otherwise, we shall consider only undirected simple graphs (i.e., any two vertices are joined by at most one edge) which are not necessarily finite and do not contain isolated vertices.

Received: August 12, 2015

Published: February 26, 2016

§ Correspondence author (c) 2016 Academic Publications, Ltd.

url: www.acadpubl.eu 
As a generalization of Harary's notion of cognitive balance in singed graphs [5], the notion of an even edge coloring of a graph $G$ was introduced by B. D. Acharya [2] as an assignment $g$ of colors to the edges of $G$ such that in every cycle of $G$ there is a nonzero even number of edges of at least one color. The minimum number of colors in an even edge coloring of $G$ will be denoted $\varepsilon(G)$.

We now define vertex analogous of even edge coloring which is generalization of consistent marked graphs (See [3]) defined as follows: An even vertex coloring is an assignment of colors to the vertices of $G$ such that in every cycle of $G$, there is a nonzero even number of vertices of at least one color. Clearly a graph need not have an even vertex coloring. For example, complete graph on 5 vertices. A graph $G$ is said to be even vertex-colorable if $G$ has an even vertex coloring.

A graph $G$ is said to be even vertex colorable, if $G$ has an even vertex coloring. The following result characterizes complete graphs which are even vertex colorable.

Theorem 1. The complete graph on $n$ vertices $K_{n}$ is even vertex colorable if, and only if, $n \leq 4$.

Proof. (Necessary) Necessary part is immediate, since, complete graph $K_{n}$, $n \leq 4$ is even vertex colorable.

(Sufficiency) Suppose that $K_{n}, n \geq 5$ is even vertex colorable and let $g$ be an even vertex colorig. Clearly $K_{n}$ contains a cycle $C$ of length 5 . By our assumption, there exists at least one color $c_{i}$ which occurs an even number of times on the cycle $C$. Suppose there are 3 vertices with color $c_{i}$, then since $G$ is complete, these three vertices form a cycle of length 3 in $G$ in which all vertices are colored $c_{i}$, a contradiction. On the other hand if there are exactly two vertices with color $c_{i}$, then $C$ contains 3 vertices which are colored by the color other than $c_{i}$. Now suppose that all the vertices does not colored same, then the cycle $C$ receives 3 colors. But a vertex from each color class forms a cycle of length 3 receiving 3 colors, a contradiction. This completes the proof.

The characterization of graphs which are even vertex colorable is not easy and so we pose the following problem:

Problem 2. Characterize even vertex colorable graphs?

Clearly, if $G$ does not contain any cycle (such a graph is often said to be 'acyclic' or called a 'forest'), then by the vacuous case of the definition, it follows that every vertex coloring of $G$ is an even vertex-coloring of $G$. More generally, if an vertex $v$ of $G$ not belonging to any cycle of $G$ then its color does not figure in the definition of an even vertex coloring of $G$ and hence it can be arbitrary. Hence, unless specified otherwise, by a 'graph' $G$, we mean a graph in which 
every vertex belongs to a cycle of $G$. Let $|g|$ denote the number of colors in an even vertex coloring $g$ of $G$ if $G$ has an even vertex coloring. The minimum number of colors in an even vertex coloring of $G$ will be denoted $\varepsilon^{v}(G)$ and a minimum even vertex-coloring of $G$ is one with exactly $\varepsilon^{v}(G)$ colors. If a $G$ is not even vertex colorable we assume $\varepsilon^{v}(G)=0$.

\section{Minimum Even Vertex Colorings}

Clearly, for any graph $G, \varepsilon^{v}(G)=1 \Longleftrightarrow G$ is bipartite, and the class of bipartite graphs is well studied in literature and a large number of their properties and uses are available in standard text-books of graph theory (e.g., see $[4,7,8]$. Hence, we consider graphs with $n=\varepsilon^{v}(G)>1$.

Further, if in an even vertex coloring $g$ of $G$, each color occurs a nonzero even number of vertices of at least one cycle in $G$, let we say that the coloring is complete and if for each vertex $v$ that is in a cycle of $G$ there is a cycle containing a nonzero even number of vertices of the color of $v$ then the coloring is said to be optimal. For each color $c_{i}$ in a minimum coloring $g$ of $G$, let the subgraph spanned by the set of vertices receiving color $c_{i}$ in $g$ be denoted $V_{i}$. Clearly, if $g$ is complete, $V_{i}$ contains at least one cycle $C$ of $G$ such that $0<\left|V(C)-V_{i}\right| \equiv 0(\bmod 2)$ and $P=\left\{V_{1}, V_{2}, \cdots, V_{n}\right\}, n=|g|$, is a partition of $V(G)$.

Theorem 3. For any graph $G$ with $\varepsilon^{v}(G) \geq 3$, any minimum even vertex coloring $g$ of $G$ is both optimal and complete.

Proof. Suppose $g$ is not optimal. Then, there is a cycle $C$ in $G$ containing an vertex $v$ such that there is no cycle in $G$ that contains a nonzero even number of edges having the color of $v$, say $g(v)=c_{i}$. Therefore, every cycle in $G$ that contains $v$ has only an odd number of vertices with color $g(v)$. Let us recolor all the edges of $G$ having the color $g(v)$ with one of the existing colors other than $g(x)$, say $c_{j}$, and let $g_{0}$ be the resulting vertex coloring of $G$. Since $g$ is an even vertex coloring of $G$ with more than three colors, every cycle containing $v$ must contain an even number of edges of some color $c_{k}$ other than $c_{i}$. Hence, suppose amongst the other cycles (that is, those which do not contain $v$ ) in $G$ there is a cycle $C_{0}$ having an vertex $u$ with color $g(v)$. Then, by our assumption, it follows that $C_{0}$ contains a nonzero even number of vertices of a color other than $g(v)$. Thus, every cycle of $\left(G, g_{0}\right)$ has a nonzero even number of vertices of at least one color, implying thereby that $g_{0}$ is indeed an even vertex coloring of $G$. This contradicts the minimality of $g$. Hence, $g$ must be optimal. 
Next, suppose $g$ is not complete. By definition, we then must have a color, say $c_{i}$, in $g$ that does not occur on a nonzero even number of vertices in any cycle of $G$. That is, if there is any vertex having color $c_{i}$ in a cycle of $G$ it must occur on an odd number of its vertices. However, since $g$ is an even vertex-coloring of $G$ each such cycle must have a nonzero even number of vertices of some color other than $c_{i}$. Hence, repainting all the vertices in $G$ having color $c_{i}$ with one of the existing colors, say $c_{j}$, other than $c_{i}$, resulting in a new vertex-coloring $g_{0}$ of $G$, does not alter their requirement to be still in consonance with the definition of an even vertex-coloring of $G$, since $\varepsilon^{v}(G) \geq 3$. But then, we have a contradiction to the minimality of $g$ again. Thus, it follows that $g$ must be complete and the theorem is proved.

Remark: The converse of the above theorem need not be true. Since any even vertex coloring of even cycle of order $>4$ satisfies both the conditions that is both optimal and complete but it is not minimal since $\varepsilon^{v}(G)=1$.

A marked graph $M=(G, \mu)$ is consistent if every cycle of $G$ is contains an even number of vertices marked - (See [3]). Clearly a marked graph in which every vertex is marked + is consistent and a marked graph in which every vertex is marked - is consistent if and only if the underlying graph is bipartite.

Theorem 4. [L. W. Beineke and F Harary[3]] If $M$ is consistent and has some negative points, then the subgraph induced by the negative points must be bipartite.

Theorem 5. [L. W. Beineke and F Harary[3]] If $M$ is consistent and point $u$ is positive while point $v$ is negative, there cannot be three disjoint $u: v$ paths.

Given an unmarked graph, it can always be marked in a consistent way by giving each vertex by a positive sign. However, this cannot always be done if some negative sign must be used. Consider for example the complete graph $K_{4}$. A graph $G$ markable if it can be consistently marked using at least one sign. Beineke and Harary [3] introduced the problem of characterizing markable graphs.

A graph $M$ is said to be markable if it is possible to mark the vertices of $G$ such that the resulting marked graph is consistent and there exists at least one vertex is marked -.

If $\varepsilon^{v}(G)=2$ and the colors are interpreted as being positive or negative then we have a marked graph in which every cycle contains a nonzero even number of positive vertices or a nonzero even number of negative vertices. We say that a marked graph $G_{\mu}$ is said to be quasiconsistant if every cycle of $G$ contains a non-zero even number of vertices marked positive or a even number 
of vertices marked negative. Clearly, a marked graph $G$ is quasiconsistant if and only if its negation $\eta(G)$ (i.e., the marked graph obtained from $G$ by reversing the sign of every vertex) is also so. There are social systems whose marked graph structures are quasi consistant and hence it would be of interest to find a structural characterization of such marked graphs. As there is no structural characterization of consistent marked graph, it is difficult to find such characterizations.

Problem 6. Characterize the quasi consistent marked graphs.

\section{Multigraph Associated with Even Vertex Coloring}

For any finite graph $G$ together with a minimum vertex coloring $g$, one can define a bipartite multigraph $B_{g}(G)$ as follows: The vertex set of $B g(G)$ has $V_{1}=\left\{c_{1}, c_{2}, \cdots, c_{n}\right\}, n=|g|$ as one partite set, where $c_{i}^{\prime} s$ are the colors by $g$ to the vertices of $G$, and $V_{2}=\left\{C^{1}, C^{2}, \cdots, C^{\mu}\right\}$ is a cycle-basis of $G$, that is, a set of $\mu$ linearly independent fundamental cycles $C_{j}, \mu=\mu(G)$ being the cyclerank of $G$. Further, the multiset $E(B g(G))$ of the edges of $B_{g}(G)$ is defined by the presence of exactly $e_{i j}$ unordered pairs $c i C j$, whenever $e_{i j}$ is the nonzero even number of vertices in $C^{j}$ having color $c_{i}$. The following rather far-fetched fact points at a natural connection between even vertex colorings and Eulerian graphs.

Theorem 7. Let $(G, g)$ be any finite graph in which every vertex belongs to a cycle together with a minimum even vertex coloring $g$. Then, the multigraph $B_{g}(G)$ is Eulerian.

Proof. Let $V_{1}=c_{1}, c_{2}, \ldots, c_{n}$ be the set of colors assigned by $g$ to the vertices of $G$ and let $V_{2}=C^{1}, C^{2}, \cdots, C^{\mu}$ is an arbitrarily fixed cycle-basis of $G$. Since every vertex of $G$ belongs to a cycle, whence $\mu(G)>0$ and $V_{1}$ and $V_{2}$ are nonempty sets. Since $g$ is an even vertex coloring of $G$, the edge $c_{i} C^{j}$ appears in $B_{g}(G)$ exactly as many times as the nonzero even number of edges in $C_{j}$ have the color $c_{i}$, the degree of the vertex $c_{i} \in V_{1}$ being a finite sum of at most $\mu(G)$ such even numbers must be even for each $i, 1 \leq i \leq n$. The same also holds for each vertex of $V_{2}$ in $B_{g}(G)$. Further, since $|g|=\varepsilon^{v}(G)$, by completeness and optimality of $g$, it follows that $B_{g}(G)$ is connected. Thus, by a well known characterization of Eulerian graphs, we see that $B_{g}(G)$ is indeed an Eulerian graph. 


\section{Acknowledgement}

The authors are thankful to the anonymous referee for valuable suggestions and comments for the improvement of the paper. Also, the first author is grateful to Dr. M. N. Channabasappa, Director and Dr. Shivakumaraiah, Principal, Siddaganga Institute of Technology, Tumkur, for their constant support and encouragement.

\section{References}

[1] B.D. Acharya, Even edge coloring of a graph, J. Combin. Theory Ser. B, 35 (1983), 78-79.

[2] B.D. Acharya, Even edge-colorings of a graph: II. A lower bound for maximum even edge-coloring index, Nat. Acad. Sci. (India) Sci.-Letters, 5, No. 3 (1982), 97-99.

[3] L. W. Beineke and F. Harary Consistant Graphs with Signed Points Rivista di Matematica per le Scienze Economiche e Sociale, 1 (1978), 81-88.

[4] G.T. Chartrand and P. Zhang, An Introduction to Graph Theory, Walter Rudin Series in Advanced Mathematics, Mc- Graw Hill Companies Inc., New York (2005).

[5] F. Harary, On the notion of balance of a signed graph, Mich. Math. J., 2 (1953), 143-146.

[6] F. Harary, R.Z. Norman and D. Cartwright, Structural Models: An Introduction to the Theory of Directed Graphs, Wiley, New York (1965).

[7] F. Harary, Graph Theory, Addison-Wesley Publ. Comp., Massachusetts, Reading (1969).

[8] D.B. West, Introduction to Graph Theory, Prentice-Hall of India Pvt. Ltd., 1999. 